\title{
Gradient and Core-Cladding Waveguides Fabrication in Porous Glass
}

\author{
Julia V. Mikhailova ${ }^{1}$, Zhong Lijing ${ }^{1,2}$, Andrey A. Ostanin ${ }^{1}$, Maksim M. Sergeev ${ }^{1}$, Roman A. Zakoldaev ${ }^{* 1}$ \\ ${ }^{1}$ ITMO University, 49, Kronverkskiy av., 197101, St. Petersburg, Russia \\ ${ }^{2}$ Huazhong University of Science \& Technology, Luoyu Road 1037, Wuhan, China \\ *Corresponding author's e-mail: zakoldaev@gmail.com
}

\begin{abstract}
We report on waveguides laser writing inside porous glass. Depending on applied laser regimes, two types of waveguides are fabricated in the same glass sample. A gradient waveguide type is achieved under the lowest pulse energy regime, which results in gradual densification of a nanoporous framework forming a core with an increased refractive index. The surrounding unirradiated material acts as a cladding. The highest pulse energy applied enables formation of the densified core surrounded by rarefaction regions in the nanoporous framework - a corecladding waveguide type. Laser light guiding is also accomplished for both types to estimate the refractive index contrast. In addition, we determine the temperature distribution based on the thermal conduction model to reveal the difference of temperature gradients for the regimes applied.
\end{abstract}

DOI: $10.2961 /$ jlmn.2020.03.2016

Keywords: femtosecond laser pulses, waveguides, porous glass, internal modification, nonlinear absorption, thermal conduction model.

\section{Introduction}

Femtosecond direct writing is a universal tool for threedimensional integration of functional structures inside glass materials $[1,2]$. Such structures as nanogratings [3], local densifications [4], submicrometer birefringent dots [5, 6] are responsible for light transformation and/or transmission in a glass chip. One of discussed examples is a waveguide [7], which is widely used as a sensor in commercial glasses. A waveguide's cladding is located close to the glass surface and responses to external physicochemical factors like temperature change [8], fluids addition [9] or light illumination [10].

In this case, the choice of a substrate is a key issue. Thus, a branched nanoporous framework of a porous glass (PG) plate is likely to bring novel abilities for storing, capturing and transferring an analyte or a reagent fore ecology needs [11] or renewable energy sources [12]. PG has been widely applied for producing optical composites [13], membranes [14] and sensors [15]. Further development of such a platform suggests laser-based fabrication of buried photonics components. A variety of structures has already been produced in PG such as waveguides in Eu-doped PG [16], multi-purpose fluidic elements [17] and microchannels [18]. However, the processing regime including the writing geometry, energy parameter, nanopores pre-filling with photo-thermosensitive components affect the result of glass structural modification. The prerequisites and possibilities of recording waveguide structures in initially pure PG were performed earlier by our group [19, 20]. Therefore, it is extremely important to evaluate and identify the types of waveguides in pure PG.

As for other dielectrics, the action of femtosecond laser pulses focused in PG induces non-linear ionization, generation of electron plasma, whose relaxation leads to a thermal source formation. Internal heat accumulation affects a nanoporous framework and activates non-reversible pore collapse resulting in material density change [20]. Depending on the temperature gradient in the irradiated zone, several types of structural modification occur. The estimation of the temperature gradient responsible for the changes mentioned is favorable to make the $\mathrm{PG}$ processing more predictable and provide technological progress.

To manage this process, the simulation of the thermal source, which is stabilized in time and space, is relevant. Miyamoto, et. al. [21, 22] proposed a numerical calculation of temperature distribution using a thermal conduction model, indicating the behavior of laser absorptivity. The calculation results agreed well with the experimental ones retrieved at glass welding $[23,24]$. Thus, it seems preferable to conduct the numerical calculation for PG to determine the fabrication regimes for various types of waveguides.

Herein, we focus on femtosecond direct writing of waveguides in PG as well as their testing. A careful calculation of the temperature distribution clarifies two types of waveguides - gradient and core-cladding.

\section{Experiment and Methods}

\subsection{Material and experimental procedures}

In this work PG samples with thickness of $1.0 \mathrm{~mm}$, average pore size $\sim 4 \mathrm{~nm}$, porosity $28 \%$ are used. The samples are fabricated through chemical etching of borosilicate glass [25]. The samples possess high transparency $(\sim 90 \%)$ in the visible and near IR ranges $(0.4-1.1 \mu \mathrm{m})$. Table 1 presents the sample physical properties required for the current research.

Table 1 Thermal and optical properties of PG sample

\begin{tabular}{cc}
\hline Parameter & Values \\
\hline Density $\rho, \mathrm{kg} / \mathrm{m}^{3}$ & 1665 \\
Thermal conductivity k, $\mathrm{W} / \mathrm{m} \cdot \mathrm{K}$ & 0.8 \\
Heat capacity $\mathrm{c}, \mathrm{J} / \mathrm{kg} \cdot \mathrm{K}$ & 1233 \\
Refractive Index $\mathrm{n}(\lambda=1030 \mathrm{~nm})$ & 1.342 \\
\hline
\end{tabular}


Femtosecond direct writing of waveguides in PG is performed on a typical laser processing station equipped with a femtosecond Yb-fiber laser (ANTAUS-20W, Avesta ltd., Russia) with the following output characteristics: pulse duration $\tau=250 \mathrm{fs}$, wavelength $\lambda=1030 \mathrm{~nm}$, output beam diameter $3.0 \mathrm{~mm}$ with the beam quality of $\mathrm{M}^{2}<1.1$, maximum pulse energy $\mathrm{E}_{\mathrm{p}}=20 \mu \mathrm{J}$ and fixed pulse repetition rate $\mathrm{f}=1 \mathrm{MHz}$; an objective $(20 \mathrm{X}, 0.4)$ and $3 \mathrm{D}$ coordinate stages based on stepper motors (Fig. 1 a).

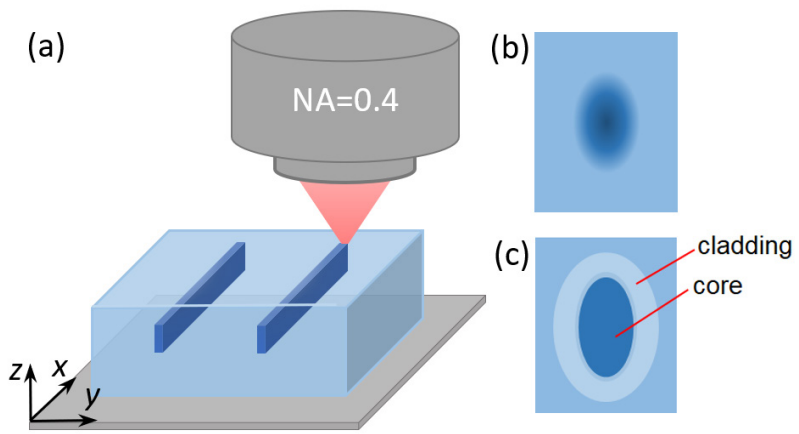

Fig. 1 (a) The schematic view of the fs-laser writing procedure. Two types of waveguides are considered in current research - (b) a gradient and (c) core-cladding.

In the first approximation, the objective provides the laser beam waist with a diameter $\left(2 \mathrm{w}_{0}\right)$ equal to $2.5 \mu \mathrm{m}$ and the Rayleigh length $\mathrm{z}_{0}=5.5 \mu \mathrm{m}$, which are defined as follows: $\omega_{0}=\mathrm{M}^{2} \lambda / \pi \mathrm{NA}$ and $\mathrm{z}_{0}=\mathrm{w}_{0}^{2} \pi / \mathrm{M}^{2} \lambda$ [26]. The applied fluence is in the range from 2.6 to $16.3 \mathrm{~J} / \mathrm{cm}^{2}$. Table 2 presents laser processing parameters. We focus on two laser regimes that enable fabrication of a gradient (Fig. $1 \mathrm{~b}$ ) and core-cladding (Fig. 1 c) waveguides.

Table 2 Laser writing parameters

\begin{tabular}{ccc}
\hline Parameters & Gradient & Core-cladding \\
\hline Pulse energy $\mathrm{E}_{\mathrm{p}}, \mu \mathrm{J}$ & $0.13-0.18$ & $0.3-0.8$ \\
Scan speed $\mathrm{v}, \mathrm{mm} / \mathrm{s}$ & $0.05-0.45$ & $0.0125-3.75$ \\
Number of pulses N & $6000-53000$ & $374-112000$ \\
\hline
\end{tabular}

After laser writing, the samples were polished at both facets to investigate the waveguide with a microscopy (Carl Zeiss) equipped with high NA objective $(50 \times, 0.65 \mathrm{NA})$. The measurement error is $3 \%$. The end-facet coupling method is applied to determine the waveguides guiding properties by capturing a near-field intensity distribution. As we showed formerly [27], He-Ne laser light couples into a waveguide by an objective, while another objective collects transmitted laser light on CCD.

\subsection{Thermal conduction model}

We focus attention on a gradient waveguide fabrication and determine experimental conditions involving a modeling step. We apply a thermal conduction model presented by Miyamoto et al. [21] to describe the thermophysical process of femtosecond laser waveguide fabrication in the PG.
In the model, the PG presents a solid-state matrix and has constant thermophysical and optical characteristics. The instantaneous source generates heat periodically in a rectangular region in the beam waist position. The source height equals the Rayleigh length $\mathrm{z}_{0}$, while the width is the beam waist diameter $(2 \omega)$. The lifetime of the source corresponds to laser pulse duration, besides the material gets cool between the pulses. The heat source is presented schematically as a rectangular solid, which uniformly moves at a constant velocity (v) along the X-axis (Fig. 2). All the constants for the calculation are given in Table 1.

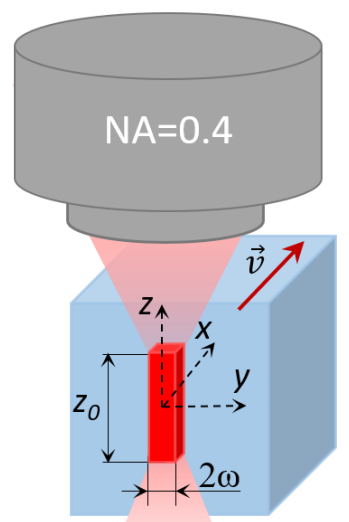

Fig. 2 Schematic representation of the heating source inside PG the rectangular solid heat source uniformly moves at a constant velocity $(v)$ along the $\mathrm{X}$-axis.

The nonlinear absorptivity is given by:

$$
A(\mathrm{z})=\frac{E_{p}}{\tau \cdot z^{2}} \cdot \frac{1}{q_{\max }},
$$

where $z$ is the distance from the center of the laser beam waist along its propagation to the Rayleigh length, $q_{\max }$ is a peak power density applied in the experiment.

The temperature in the PG sample is calculated as follows:

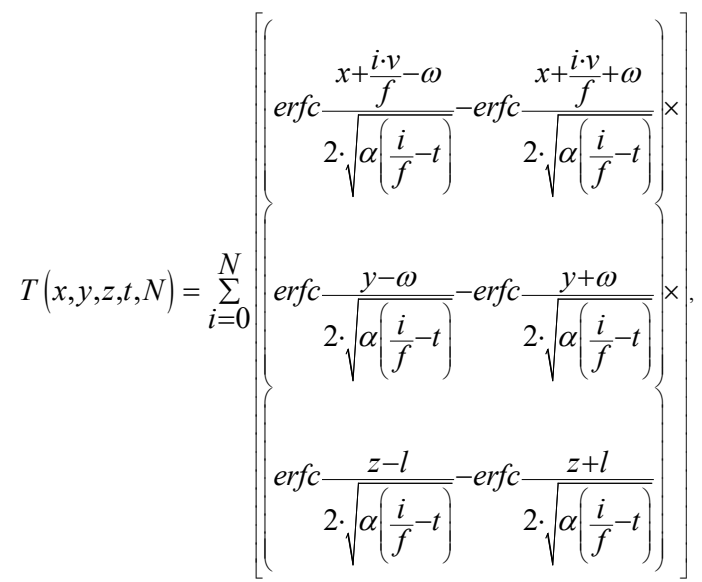

where $\alpha$ is the thermal diffusivity of the PG sample. Thus, the temperature change is:

$$
d T(x, y, z, t, N)=\frac{A(z) \cdot E_{p}}{64 \cdot c \cdot \rho \cdot \omega^{2} \cdot l} T(x, y, z, t, N)+T_{0},
$$

where $T_{0}=300 \mathrm{~K}$. 


\section{Results and discussion}

\subsection{Waveguides laser writing}

Applying different ranges of laser processing parameters pointed in Table 2, both the gradient (Fig. 3 a) and corecladding (Fig. 3 b) waveguides were fabricated in the same PG sample. The waveguides are located at the depth of 250 $\mu \mathrm{m}$ below the PG surface. The top view microscopy photo of the gradient type (Fig. 3 a) indicates a smooth modification of PG refractive index. We can see only a single-line track with a lateral size about $2.8 \mu \mathrm{m}$ and surrounded heat affected zone $\sim 6.6 \mu \mathrm{m}$. The cross-section photo of the waveguide shows a uniform glass modification and the absence of any micro-cavities inside or surrounding the track (Fig. 3 b). The height of the waveguide equals 30 $\mu \mathrm{m}$.

We can assume that nonlinear absorption causes the nanoporous matrix to heat up to the temperature, which provides a local stress increase that looks like a material density change [20]. However, it is important to avoid the action of the elastic stresses, as they can return the material to its original state after finishing the laser writing step. Thus, the relaxation of elastic stresses is an integral part for the regime of waveguides fabrication. In this case, the action of thermomechanical stresses is preferred and achieved in the regime of plasma generation with a corresponding shock wave action. Hydrodynamic shock waves occur under laser irradiation and propagate from the center of the laser beam waist to its periphery, where the temperature is significantly lower [28, 29]. This leads to material rarefaction and densification. Such structural changes are nanoscale and cannot be revealed by the optical microscopy.

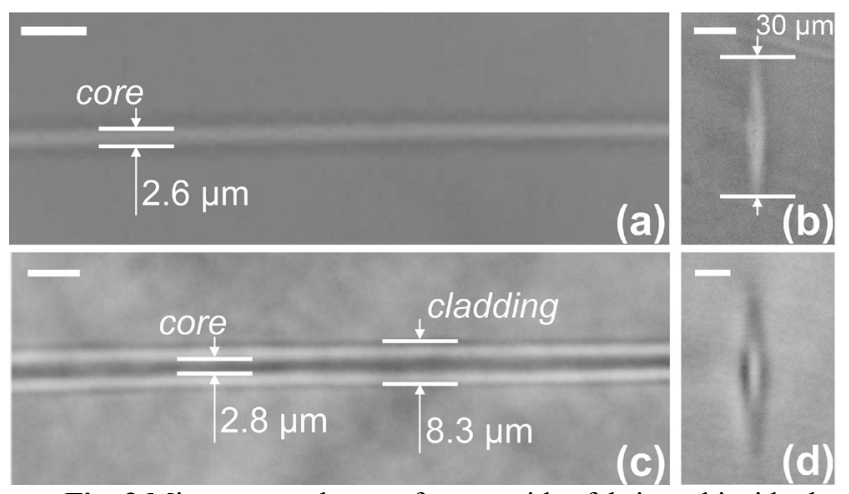

Fig. 3 Microscopy photos of waveguides fabricated inside the PG plate by femtosecond laser pulses: the gradient $(\mathrm{a}, \mathrm{c})$ and corecladding $(\mathrm{c}, \mathrm{d})$ types, where $(\mathrm{a}, \mathrm{c})$ its top view and $(\mathrm{c}, \mathrm{d})$ are sideview The scale bars equal $10 \pm 0.3 \mu \mathrm{m}$.

At the same time, increasing pulse energy enables formation of the core-cladding type consisting of several layers with different density across the writing direction (Fig. $3 \mathrm{c}, \mathrm{d})$. According to microphotos the waveguide core size equals $2.8 \mu \mathrm{m}$ and cladding $8.3 \mu \mathrm{m}$. The waveguide of this type is about three times wider than the previous one.

\subsection{Waveguides coupling}

Laser light coupling with waveguides demonstrates the real structure of the waveguide fabricated. First, we captured the near-field intensity distribution for the both waveguide types (Fig. 4 a,b). The elliptical shape accompanies the gradient waveguide due to its geometry (Fig. 3 b). A smaller aspect ratio was obtained for the second type waveguide. In general, both of them show satisfactory intensity distribution - a single maximum. The aspect ratio, associated with the shape of the waveguide, depends on the laser writing conditions. It is important to note that there are no difficulties to create a symmetric waveguide [30]. The current research avoids the use of any diffractive components in the optical system and prefers to maintain the initial Gaussian beam for laser processing.

The profile of a refractive index contrast $(\Delta \mathrm{n})$ in the cross-section of the waveguides from the near-field distribution was also estimated by solving the HelmholtzEquation [31] that demonstrates the relationship between the refractive index profile and near-field distribution of waveguide output (Fig. 4 c,d). An improved algorithm proposed by Mansour [32] was adopted for the calculations. The refractive index profile of the gradient waveguide shows acceptable conditions, where a gradual change in the contrast from the center to the periphery is noticeable. In addition, there is no negative index change. These facts prove the gradient type of the waveguide. The refractive index contrast $(\Delta \mathrm{n})$ equals $\sim 5.0 \cdot 10^{-4}$. As for the corecladding type, there is a highly depressed cladding with $\Delta \mathrm{n}$ of $\sim 1.0 \cdot 10^{-3}$ surrounding the core. That maximum value of $\Delta \mathrm{n}$ between core and cladding obviously increases up to $\sim 1.8 \cdot 10^{-3}$

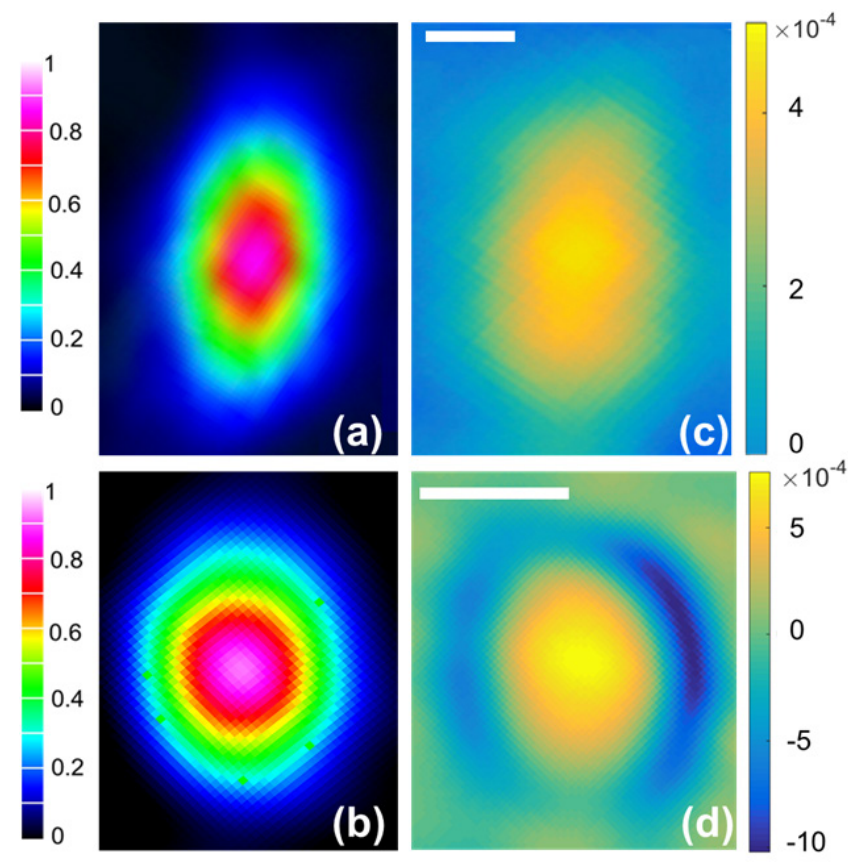

Fig. 4 Waveguides testing results. The captured near-field distribution at $632.8 \mathrm{~nm}$ for (a) the gradient waveguide written by $0.18 \mu \mathrm{J}$ pulse energy at translation speed of $0.45 \mathrm{~mm} / \mathrm{s}$ and (b) core-cladding waveguide written by $0.8 \mu \mathrm{J}$ pulse energy at translation speed of $1.25 \mathrm{~mm} / \mathrm{s}$. (c,d) The corresponding refractive index contrast for the waveguides. The scale bars equal $5 \mu \mathrm{m}$.

\subsection{Temperature distribution in affected area}

Thermophysical modeling simulates the temperature distribution in the laser irradiated area and its periphery. Figure 5 demonstrates the top view and 3D temperature distributions for both types of the waveguides - gradient and 
core-cladding. The calculation is accomplished in MathCAD software considering experimental regimes (for the gradient waveguide $\mathrm{E}_{\mathrm{p}}=0.18 \mu \mathrm{J}, v=0.45 \mathrm{~mm} / \mathrm{s}$, for core-cladding waveguide $\mathrm{E}_{\mathrm{p}}=0.8 \mu \mathrm{J}, v=1.25 \mathrm{~mm} / \mathrm{s}$ ) for $0.99 \mu$ s after the waveguide fabrication. A good agreement of experimentally measured waveguides lateral sizes with calculated temperature fields distribution is confirmed. According to the simulation, the gradient waveguide regime results in the temperature rising up to $1450 \mathrm{~K}$, which is significant for nanoporous framework densification [33]. However, the core-cladding regime leads to much higher temperatures with the peak over $10100 \mathrm{~K}$. As previously mentioned, under the laser irradiation the shock waves occur and lead to local material structural changes. The nature of these changes can be associated with the pulse energy and the gradient of the temperature distribution in the laser irradiated area and its periphery.

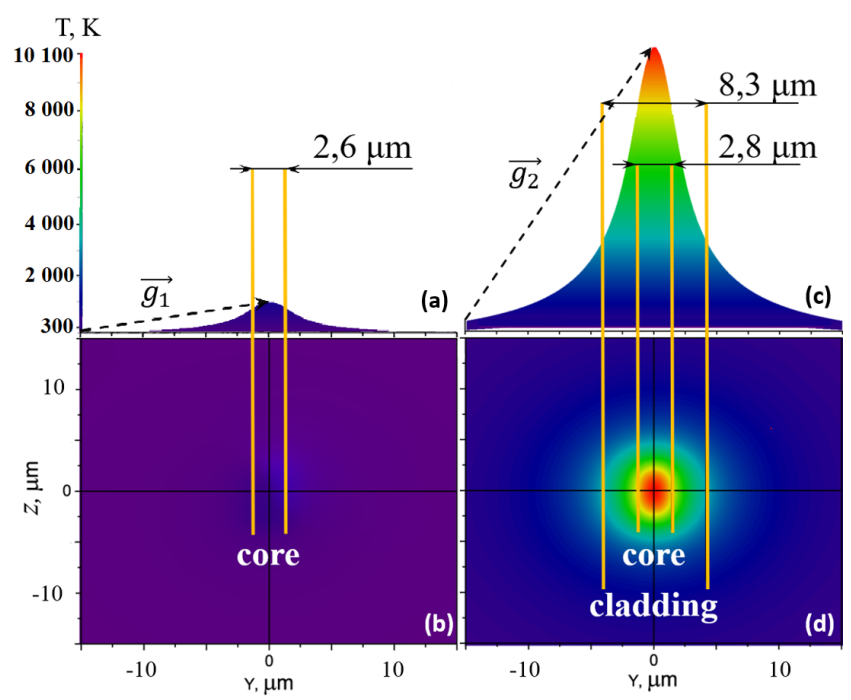

Fig. 5 Simulation results of temperature distribution inside PG irradiated by femtosecond laser in two regimes for the gradient $(\mathrm{a}, \mathrm{b})$ and core-cladding $(\mathrm{c}, \mathrm{d})$ waveguides. The distribution was calculated for $0.99 \mu$ s after the waveguides fabrication.

To clarify the difference between regimes, we estimated the temperature gradient via calculating the magnitudes of gradient vectors $\left(g_{1}\right)$ and $\left(g_{2}\right)$ (Fig. 5 a,c). These vectors are directed from the initial point on the edge of simulated temperature distribution to the peak point in the center of the laser irradiated area. The magnitude of vector $\mathbf{g}_{1}$ equals $1 \cdot 10^{-3}$ while $\mathrm{g}_{2}=9 \cdot 10^{-3}$. Therefore, a significantly higher temperature gradient for the core-cladding regime rather than for the gradient one formed two areas in the PG sample under femtosecond laser irradiation.

The low gradient value (Fig. 5 a) leads to the gradient regime characterized with the refractive index smoothly increasing from the periphery to the center of the laser irradiated area (Fig. 4 b). For the core-cladding regime, in the irradiated area the temperature gradient is significantly higher (Fig. 5 c). It forms a clearly defined region of material densification with the increased refractive index surrounded with the rarefaction region (Fig. $4 \mathrm{~d}$ ).

\section{Conclusions}

In conclusion, we have demonstrated the laser direct writing of two types of waveguides in PG - the gradient and core-cladding. The main difference of the second type is the presence of a rarefaction region surrounding the core. Meanwhile, both types have a core lateral size in the range $2.6-8.3 \mu \mathrm{m}$.

Both types of waveguides have broad application prospects. For example, gradient waveguides can be attractive for sensor applications as they are sensitive to molecules captured by the nanoporous framework. The corecladding waveguides are also sensitive to fluids accumulated in the surrounding rarefaction regions. An additional advantage of this type is an increased refractive index contrast that is important for compact waveguides location in a single glass sample.

A careful calculation of thermal field distribution based on the thermal conduction model shows the gradient waveguide to be associated with low temperature gradient with maximum temperature corresponding to nanoporous framework densification $(\sim 1400 \mathrm{~K})$. As a result, this regime enables formation of a smooth densification inside the sample. Increasing the pulse energy results in broadening of the thermal field with the maximum temperature of $10100 \mathrm{~K}$. In our experiment this significant temperature gradient change led to the rarefaction region surrounding the densified core as well as increased the waveguide lateral size several times. Thus, thermal conduction modeling contributes to the choice of the waveguide fabrication regime in $\mathrm{PG}$.

\section{Acknowledgments}

The study is funded by the grant of Russian Science Foundation (project No. 19-79-10208).

\section{References}

[1] K. Sugioka: Nanophotonics, 6, (2017) 393.

[2] S. Lei, X. Zhao, X. Yu, A. Hu, S. Vukelic, M.B. Jun, H.E. Joe, Y.L. Yao, and Y.C. Shin: J. Manuf Sci Eng, 142, (2020) 031005-1.

[3] A. Rudenko, J.-P. Colombier, and T. Itina: Phys. Chem. Chem. Phys, 8, (2018) 5887.

[4] Y. Bellouard, A. Champion, B. McMillen, S. Mukherjee, R.R. Thomson, C. Pépin, P. Gillet, and Y. Cheng: Optica, 3, (2016) 1285.

[5] S. Fedotov, A. Okhrimchuk, A. Lipatiev, A. Stepko, K. Piyanzina, G.Y. Shakhgildyan, M.Y. Presniakov, I. Glebov, S. Lotarev, and V. Sigaev: Opt. Lett., 43, (2018) 851.

[6] A.S. Lipatiev, S.S. Fedotov, A.G. Okhrimchuk, S.V. Lotarev, A.M. Vasetsky, A.A. Stepko, G.Y. Shakhgildyan, K.I. Piyanzina, I.S. Glebov, and V.N. Sigaev: Appl. Opt., 57, (2018) 978.

[7] K.M. Davis, K. Miura, N. Sugimoto, and K. Hirao: Opt. Lett., 21, (1996) 1729.

[8] J. Lapointe, M. Gagné, M.-J. Li, and R. Kashyap: Opt. Express, 22, (2014) 15473.

[9] J. Lapointe, F. Parent, E.S. de Lima Filho, S. Loranger, and R. Kashyap: Opt. Lett., 40, (2015) 5654. 
[10] A. Abou Khalil, J.-P. Bérubé, S. Danto, J.-C. Desmoulin, T. Cardinal, Y. Petit, R. Vallée, and L. Canioni: Sci. Rep., 7, (2017) 1.

[11] T.A. Tsyganova and T.V. Antropova: J. Phys. Conf. Ser., 1134.1, (2018) 012063.

[12] H. Mögelin, G. Yao, G. Zhong, A.R. dos Santos, A. Barascu, R. Meyer, and T. Turek: J. Power Sources, 377, (2018) 18

[13] L. Ermakova, A. Kuznetsova, T. Antropova, A. Volkova, and I. Anfimova: Colloid J., 82, (2020) 262.

[14] D. Lebedev, M. Novomlinsky, V. Kochemirovsky, I. Ryzhkov, I. Anfimova, M. Panov, and T. Antropova: Materials, 13, (2020) 1767.

[15] K. Izumi, M. Utiyama, and Y.Y. Maruo: Sens. Actuators B Chem., 268, (2018) 1.

[16] V. N. Bagratashvili, E. A. Chutko, V. M. Gordienko, I. A. Makarov, and M. A. Timofeev: Laser Phys. Lett., 5, (2008) 671.

[17] V.P. Veiko, R.A. Zakoldaev, M.M. Sergeev, P.A. Danilov, S.I. Kudryashov, G.K. Kostiuk, A.N. Sivers, A.A. Ionin, T.V. Antropova, and O.S. Medvedev: Opt. Express, 21, (2018) 28150.

[18] Yang Liao, Yongfeng Ju, Long Zhang, Fei He, Qiang Zhang, Yinglong Shen, Danping Chen, Ya Cheng, Zhizhan Xu, Koji Sugioka, and Katsumi Midorikawa: Opt. Lett., 19, (2010) 3225.

[19] V.P. Veiko, S.I. Kudryashov, M.M. Sergeev, R.A. Zakoldaev, P.A. Danilov, A.A. Ionin, T.V. Antropova, and I.N. Anfimova: Laser Phys. Lett., 5, (2016) 055901.

[20] T.E. Itina, R.A. Zakoldaev, M.M. Sergeev, H. Ma, S.I. Kudryashov, O.S. Medvedev, and V.P. Veiko: Opt. Mater. Express, 9, (2019) 4379.

[21] I. Miyamoto, K. Cvecek, and M. Schmidt: Opt. Express, 19, (2011) 10714.

[22] I. Miyamoto, K. Cvecek, Y. Okamoto, and M. Schmidt: Appl. Phys. A, 114, (2014) 187.

[23] I. Miyamoto, A. Horn, J. Gottmann, D. Wortmann, and F. Yoshino: J. Laser Micro/Nanoengin., 2, (2007) 57.

[24] I. Miyamoto, K. Cvecek, and M. Schmidt: Opt. Express, 21, (2013) 14291.

[25] O. Andreeva and O. Bandyuk: Holograms-Recording Materials and Applications, (2011) 45.

[26] T. T. Fernandez, M. Sakakura, S. M. Eaton, B. Sotillo, J. Siegel, J. Solis, Y. Shimotsuma, and K. Miura: Prog. Mater. Sci., 94, (2018) 68.

[27] L. Zhong, R.A. Zakoldaev, M.M. Sergeev, V.P. Veiko, and Z. Li: Opt. Quantum Electron., 52, (2020) p.49.

[28] D.Y. Tzou and E.J. Pfautsch: J. Eng. Math., 61, (2008) 231.

[29] Z. Zhang and G. Gogos: Phys. Rev. B, 69, (2004) 235403.

[30] M. Ams, G. Marshall, D. Spence, and M. Withford: Opt. Express, 13, (2005) 5676.

[31] A. Szameit, J. Burghoff, T. Pertsch, S. Nolte, A. Tünnermann, and F. Lederer: Opt. Express, 14, (2006) 6055.

[32] I. Mansour and F. Caccavale: J. Lightwave Technol., 14, (1996) 423.

[33] T.V. Antropova, I.A. Drozdova, T.N. Vasilevskaya, A.V. Volkova, L.E. Ermakova, and M.P. Sidorova: Glass Phys. Chem., 33, (2007) 109. 